\title{
Coronavirus disease 2019 (COVID-19) in older patients: outcomes and risks of mortality
}

Christopher Nabors, Abhinaya Sridhar, Wilbert S. Aronow, Urvashi Hooda, Lawrence Huang, Merita Shehu, Melissa Gennarelli, Stephen A. Lobo, Abhay Dhand

Department of Medicine, Westchester Medical Center, Valhalla, US

Submitted: 4 September 2021

Accepted: 13 December 2021

Arch Med Sci Aging 2021; 4: e33-e41

DOI: https://doi.org/10.5114/amsa.2021.112472

Copyright (c) 2021 Termedia \& Banach

\begin{abstract}
Introduction: To understand how age and other factors impacted outcomes, we examined characteristics of patients aged 65 years and older hospitalized with COVID-19.

Material and methods: This was a retrospective cohort study that included all patients aged 65 years and older with laboratory-confirmed COVID-19, who were admitted to a suburban New York academic medical centre between 15 March and 13 May 2020, and discharged.

Results: Of 196 patients, the median age was 76 years, with 57\% male, and $66 \%$ white. A greater proportion of "older" (77-105 years) compared to "old" (65-76 years) patients were admitted with a primary diagnosis other than COVID-19 (34\% vs. $15 \%$ ), were afebrile ( $80 \%$ vs. $67 \%$ ), and had a clear initial chest X-ray ( $19 \%$ vs. $8 \%)$. Older patients had a higher prevalence of dementia ( $26 \%$ vs. $1.0 \%$ ), cardiac ( $42 \%$ vs. $28 \%$ ), and vascular disease $(20 \%$ vs. $9 \%)$. Overall survival was lower among older compared to old patients ( $55 \%$ vs. $74 \%, p=0.026)$ and when mechanical ventilation $(20 \%$ vs. $46 \%$, $p=0.29)$ or vasopressors ( $15 \%$ vs. $41 \%, p=0.46)$ were required $(20 \%$ vs. $46 \%, p=0.029)$ and when new hepatic dysfunction ( $24 \%$ vs. $65 \%, p<0.001)$ or new renal failure ( $29 \%$ vs. $56 \%, p=0.015)$ developed. Factors at presentation that were associated with significantly lower survival included hypoxaemia, elevation of total white blood cell count, procalcitonin, and d-dimer. Conclusions: Overall mortality was $34 \%$. Survival was 2 - to 3 -fold higher for those aged 65-76 years compared to those aged 77 years and older who required advanced therapies such as mechanical ventilation. Improving clinical parameters were associated with significantly higher survival, regardless of age.
\end{abstract}

Key words: COVID-19, coronavirus, aging, pandemic.

\section{Introduction}

Patients of advanced age who are hospitalized with Coronavirus disease 2019 (COVID-19) have a higher risk of hospitalization, morbidity, greater need for a higher level of care, and higher mortality when compared to younger patients. Among 5700 patients hospitalized with COVID-19 in New York, early in the pandemic, mortality was around 43\% for those aged 70 years [1]. A large, single-centre study from the western US showed a mortality rate of $37 \%$ among patients aged 80 years or older hospitalized with COVID-19 [2]. Previously published studies of COVID-19 in older patients (> 65 years) do not adequately explain the

\author{
Corresponding author: \\ Christopher Nabors \\ Department of Medicine \\ Westchester Medical Center \\ Valhalla, United States \\ Phone: 914-493-5127 \\ E-mail: ccnabors@gmail.com
}


heterogeneity in outcomes related to age subgroups nor the association of age-related conditions such as immune senescence, frailty, and the prevalence of particular chronic comorbid conditions with unfavourable outcomes [1, 3, 4]. For that reason, we examined the clinical course and characteristics of patients aged 65 years and older who were hospitalized with COVID-19 at a suburban tertiary care centre during the early pandemic.

\section{Material and methods}

This is a retrospective cohort study conducted at Westchester Medical Centre, which serves a population of 2.5 million people in the Hudson Valley region of New York State. All hospitalized patients aged 65 years or older from 15 March to 15 May 2020, who tested positive for SARSCoV-2 by polymerase chain reaction, and were discharged, were included in this study. Clinical characteristics, treatment, and hospital course were recorded, and variables were compared using Student's $t$-test, $\chi^{2}$, and/or Fisher's exact test and binary logistic regression. The primary analysis focused on 2 age groups: "Old" (65-76 years) and "Older" (77-105 years). These age groups were selected because the 76-year median age corresponded to the age at which mortality has been shown to rise rapidly in other studies $[1,5]$. Statistical significance was accepted at $p<0.05$ and corrected using the Bonferroni method.

\section{Results}

The study included 196 patients, which comprised $32 \%$ of all COVID-19 patients hospitalized during the study period. The median age was 76 years (range: 65-105 years). The majority (70\%) of patients arrived from home. The primary diagnosis at time of hospitalization was COVID-19 for $65 \%$ of patients and another diagnosis with COVID-19 for the remainder of the group. Atypical clinical presentations were common and included generalized weakness (36\%), altered mental status (18\%), myalgia (15\%), and falls (7\%), along with typical symptoms such as shortness of breath $(63 \%)$ or fever (54\%). The mean duration from symptom onset to arrival was $5.6 \pm 4$.4 days. On admission, $70 \%$ of patients had bilateral infiltrates on chest X-ray, $27 \%$ were febrile, $33 \%$ had a respiratory rate over 24 , and $53 \%$ had a lowest measured oxygen saturation level below $90 \%$. Patients had multiple underlying chronic medical conditions, with a mean of $3.9 \pm 1.9$ medical comorbidities. Common medical comorbidities were hypertension (63\%), cardiac conditions (34\%), diabetes mellitus (34\%), and neurological disorders (inclusive of dementia) (19\%). Mean body mass index (BMI) was $27.2 \pm 5.7 \mathrm{~kg} / \mathrm{m}^{2}$ (range:
14.5-44.1). Overall survival was $66.3 \%$ and did not differ significantly across various age groups 65-69 (30/40, 75\%), 70-74 (37/52, 71\%), 75-79 (24/31, $77 \%)$, but it was significantly lower in the age group 80 years and above $(39 / 73,53 \%$, $\left.\chi^{2}=9.0, p=0.003\right)$ (Table I).

\section{Comparison of age groups (65-76 "old" vs. 77-105 years "older")}

\section{Presentation}

On admission, a greater proportion of older patients arrived from a location other than home (60\% vs. $12 \%, p<0.001)$ and had an admission diagnosis other than COVID-19 (34\% vs. $15 \%$, $p=0.001)$. More older patients had a history of dementia ( $25 \%$ vs. $1.0 \%, p<0.001)$, chronic heart disease ( $42 \%$ vs. $28 \%, p=0.038$ ), a vascular disor$\operatorname{der}(20 \%$ vs. $9 \%, p=0.023)$, or recurrent bacterial infections ( $7 \%$ vs. $1.0 \%, p=0.022$ ). Significant differences in the average number of comorbidities per patient and the proportions of other comorbid conditions were not observed between the 2 age groups. Antipsychotic (14\% vs. 5\%, $p=0.031)$ and antidepressant (20\% vs. 9\%, $p=0.023$ ) drug use was reported more commonly in the older group. Mean body mass was significantly lower in the older group ( $26.0 \pm 5.3$, vs. $28.4 \pm 5.8, p=0.007)$. Clinical presentations varied significantly between the 2 groups, with a higher proportion of older patients being afebrile (33\% vs. $21 \%, p=0.047$ ), having altered mentation ( $30 \%$ vs. $7 \%, p<0.001$ ), a need for oxygen by non-rebreather mask $(27 \%$ vs. $8.0 \%, p=0.001)$, having a clear initial chest X-ray (19\% vs. $8 \%, p=0.029)$, and reliance on surrogate decision-making ( $64 \%$ vs. $21 \%, p<0.001)$.

\section{Hospital course}

The proportions of old versus older patients who had ever utilized intensive care, mechanical ventilation, vasopressors, or renal replacement therapy did not differ significantly during their hospitalization. However, survival was significantly lower for those in the older age group who required mechanical ventilation $(20 \%$ vs. $46 \%$, $p=0.029)$, vasopressors ( $15 \%$ vs. $41 \%, p=0.046)$, had new renal failure ( $29 \%$ vs. $56 \%, p=0.015)$, or new hepatic dysfunction ( $24 \%$ vs. $65 \%, p<0.001$ ). On admission, $94 \%$ of old patients and $70 \%$ of older patients placed no restrictions on potential resuscitative measures. During hospitalization, older patients were more likely than younger patients to change their wishes for cardiopulmonary resuscitation (18\% vs. $12 \%$ ). The average number of individual COVID-19-directed medication doses given to older patients was significantly lower than for old patients ( $7 \pm 9$ vs. $16 \pm 15$, $p<0.001$ ) with fewer older patients receiving azi- 
Table I. Baseline characteristics of patients hospitalized with COVID-19 aged 65 years and older

\begin{tabular}{|c|c|c|c|}
\hline \multicolumn{2}{|l|}{ Parameter } & $N$ & $\%$ \\
\hline \multicolumn{2}{|l|}{ Demographic information: } & 196 & \\
\hline \multicolumn{2}{|l|}{ Overall survival } & 130 & 66.3 \\
\hline \multicolumn{2}{|l|}{ Current age [years] (median, min./max.) } & \multicolumn{2}{|c|}{$76(65-105)$} \\
\hline \multirow[t]{2}{*}{ Sex } & $\mathrm{F}$ & 85 & 43.4 \\
\hline & M & 111 & 56.6 \\
\hline \multirow[t]{6}{*}{ Race and ethnicity $(n=194)$} & Asian/Pacific Islander & 8 & 4.1 \\
\hline & Black & 21 & 10.8 \\
\hline & Hispanic & 32 & 16.5 \\
\hline & Other/not identified & 5 & 2.6 \\
\hline & White & 127 & 65.5 \\
\hline & Native American & 1 & 0.5 \\
\hline \multicolumn{2}{|l|}{ Advanced directives and presentation: } & 196 & \\
\hline \multirow[t]{2}{*}{ Resuscitation wishes on admission } & DNR/DNI & 23 & 11.7 \\
\hline & Full measures & 173 & 88.3 \\
\hline \multirow[t]{4}{*}{ Decision-maker on admission } & Not identified & 2 & 1.0 \\
\hline & HCP/Surrogate & 76 & 38.8 \\
\hline & Patient & 117 & 59.7 \\
\hline & Physician on patient's behalf & 1 & 0.5 \\
\hline \multicolumn{2}{|c|}{ Change in resuscitation wishes during hospital course } & 29 & 14.8 \\
\hline \multirow[t]{3}{*}{ Primary diagnosis $(n=195)$} & COVID-19 & 128 & 65.6 \\
\hline & COVID-19 and other & 20 & 10.3 \\
\hline & Other condition and COVID-19 & 47 & 24.1 \\
\hline \multirow[t]{2}{*}{ Arrived from $(n=196)$} & Home & 128 & 65.3 \\
\hline & $\mathrm{NH}$, Rehab, other & 68 & 34.7 \\
\hline \multirow[t]{4}{*}{ Initial disposition $(n=196)$} & Not known & 9 & 4.6 \\
\hline & Discharged from Emergency Dept. & 9 & 4.6 \\
\hline & General Medical Ward & 134 & 68.4 \\
\hline & Intensive Care & 44 & 22.4 \\
\hline \multicolumn{4}{|l|}{ Symptoms $(n=196)$ : } \\
\hline \multicolumn{2}{|c|}{ Mean duration between symptom onset and admission [days] (SD) } & \multicolumn{2}{|c|}{$5.6(4.4)$} \\
\hline \multicolumn{2}{|l|}{ Shortness of breath } & 124 & 63.3 \\
\hline \multicolumn{2}{|l|}{ Fever } & 105 & 53.6 \\
\hline \multicolumn{2}{|l|}{ Cough } & 103 & 52.6 \\
\hline \multicolumn{2}{|l|}{ Generalized weakness } & 71 & 36.2 \\
\hline \multicolumn{2}{|l|}{ Decreased appetite (anorexia) } & 37 & 18.9 \\
\hline \multicolumn{2}{|l|}{ Altered mental status (delirium) } & 35 & 17.9 \\
\hline \multicolumn{2}{|l|}{ Diarrhoea } & 32 & 16.3 \\
\hline Myalgia & & 29 & 14.8 \\
\hline Fatigue & & 28 & 14.3 \\
\hline Fall & & 13 & 6.6 \\
\hline Medical comorbidities: & & 196 & \\
\hline Total comorbidities per patient (me & & & \\
\hline Hypertension & & 124 & 63.3 \\
\hline Diabetes Mellitus & & 66 & 33.7 \\
\hline Obesity & & 42 & 21.4 \\
\hline Chronic heart disease (CHF, CAD, arr & nia, implantable defibrillator, pacer) & 67 & 34.2 \\
\hline
\end{tabular}


Table I. Cont.

\begin{tabular}{|llc|}
\hline Parameter & $\boldsymbol{N}$ & $\%$ \\
\hline Neurological disorders: any & 37 & 18.9 \\
\hline Chronic lung disease & 30 & 15.3 \\
\hline Vascular disease: ischaemic stroke, PAD, PVD & 28 & 14.3 \\
\hline Hypothyroidism & 28 & 14.3 \\
\hline Dementia & 25 & 12.8 \\
\hline Smoker (ever) & 23 & 11.7 \\
\hline Chronic kidney disease & 18 & 9.2 \\
\hline Malignancy (any) & 17 & 8.7 \\
\hline Depression & 16 & 8.2 \\
\hline Immunosuppression & 13 & 6.6 \\
\hline Recurrent serious bacterial infections & 8 & 4.1 \\
\hline Haemodialysis & 6 & 3.1 \\
\hline Chronic liver disease & 5 & 2.6 \\
\hline
\end{tabular}

CHF - congestive heart failure, CAD - coronary artery disease, pacer - cardiac pacemaker, $\mathrm{NH}$ - skilled nursing facility, PAD - peripheral arterial disease, $P V D$ - peripheral vascular disease, SD - standard deviation from mean, race and ethnicity reported in categories based on those of the Agency for Health Care Research and Quality

thromycin $(54 \%$ vs. $78 \%, p<0.001)$, hydroxychloroquine ( $56 \%$ vs. $82 \%, p<0.001)$, non-pulse dose steroids ( $25 \%$ vs. $43 \%, p=0.006)$, or tocilizumab ( $2 \%$ vs. $14 \%, p=0.003$ ). However, the proportion of old vs. older patients who had ever received at least 1 dose of a COVID-19-directed medication was not significantly different (Table II).

\section{Outcomes}

Overall survival for the cohort was $66 \%$, but it was significantly lower (59\% vs. $74 \%, p=0.026$ ) for the older group (77-105 years) compared to the old group (65-76 years). The mean duration of hospitalization (14.1 vs. 13.5, $p=0.754$ ), mean BMI (27.21 vs. $27.23 \mathrm{~kg} / \mathrm{m}^{2}, p=0.984$ ), proportions of survivors versus non-survivors with a primary diagnosis of COVID-19 on admission (67\% vs. $33 \%, p=0.975)$, male sex $(67 \% \vee 33 \%, p=$ $0.908)$, or of different racial/ethnic groups did not differ significantly between the older/old groups. However, survival was significantly higher among older compared to old patients with BMI less than 23 (63\% vs. $38 \%, p=0.033)$. Survival was higher among those with no initial restrictions on resuscitation (69\% vs. $31 \%, p=0.014)$ and for those whose resuscitation wishes did not change during hospitalization $(73 \%$ vs. $27 \%, p<0.001)$. We did not observe significant differences in survival between patients treated versus not treated with azithromycin (64\% vs. $36 \%, p=0.102)$, hydroxychloroquine (68 vs. $32 \%, p=0.482$ ), tocilizumab (63\% vs. $38 \%, p=0.605)$, or convalescent plasma (64\% vs. $36 \%, p=0.761)$. However, survival was lower among those treated with steroids (nonpulse dose, $55 \%$ vs. $45 \%, p=0.018$ ) or pulse dose (38\% vs. $62 \%, p<0.001)$.
Factors associated with significantly higher mortality in a univariate analysis included older age group ( $59 \%$ vs. $41 \%, p=0.026$ ), pre-admission use of antidepressants, or presence of delirium or dementia on admission and the following admission parameters: oxygen saturation < $90 \%(68 \%$ vs. $46 \%, p=0.004)$, elevated procalcitonin $(64 \%$ vs. $16 \%, p<0.001)$, elevated aspartate transaminase (AST) $\geq 2 \times$ upper limit of normal (39\% vs. $17 \%, p=0.001)$, elevated total white blood count (46\% vs. $13 \%, p<0.001$ ), and elevated D-dimer $\geq 5 \times$ upper normal ( $54 \%$ vs. $20 \%, p<0.001$ ). The prevalence of these factors by incremental 5-year age subgroups and by age group 80 years and older is displayed in Figure 1. In a multivariable regression model that included each of these factors, elevated procalcitonin (odds ratio $=9.8$, 95\% Cl: 2.4-40), D-dimer (odds ratio $=4.2$, 95\% Cl: 1.1-16), and total white cell count (odds ratio $=5.3,95 \% \mathrm{Cl}: 1.3-21)$ were significantly associated with mortality (Table III). During hospitalisation, the proportion of patients who survived versus died was significantly higher among those with improving organ function: oxygenation (98\% vs. $3 \%, p<0.001)$, renal function ( $89 \%$ vs. $11 \%$, $p<0.001)$, hepatic function ( $84 \%$ vs. $16 \%, p<$ 0.001 ), or delirium ( $95 \%$ vs. $5 \%, p<0.001$ ) (Supplementary Table I).

\section{Discussion}

In this report, we provide a detailed description of characteristics and clinical outcomes in patients aged 65 years and older hospitalized with COVID-19, with further sub-group analysis among 2 different chronological age groups. In this early-pandemic cohort, overall survival was $66 \%$ 
Table II. Presenting findings, vitals, and advance directives, treatments, and outcomes (total $N=196$ )

\begin{tabular}{|c|c|c|c|c|c|c|c|}
\hline \multirow[t]{2}{*}{ Parameter } & & \multirow[t]{2}{*}{$N$} & \multicolumn{2}{|c|}{ Age 65 to 76 years } & \multicolumn{3}{|c|}{ Age 77 years and older } \\
\hline & & & $N=102$ & $\%$ & $N=94$ & $\%$ & $P$-value \\
\hline \multicolumn{2}{|c|}{ Survival (overall) Lived } & & 75 & 73.5 & 55 & 58.5 & 0.026 \\
\hline \multirow{3}{*}{$\begin{array}{l}\text { Primary } \\
\text { admitting } \\
\text { diagnosis }\end{array}$} & COVID & \multirow[t]{3}{*}{195} & 75 & 73.5 & 53 & 57.0 & 0.015 \\
\hline & COVID and other & & 12 & 11.8 & 8 & 8.6 & - \\
\hline & Other and COVID & & 15 & 14.7 & 32 & 34.4 & 0.001 \\
\hline \multirow{4}{*}{$\begin{array}{l}\text { Goals of care } \\
\text { (on admission) }\end{array}$} & Comfort only & & 0 & 0.0 & 4 & 4.3 & \\
\hline & Full care & & 96 & 94.1 & 66 & 70.2 & $<0.001$ \\
\hline & Some limits & & 3 & 2.9 & 16 & 17.0 & 0.001 \\
\hline & Some limits then comfort only & & 1 & 1.0 & 8 & 8.5 & 0.012 \\
\hline \multicolumn{2}{|c|}{ Change of resuscitation status and goals of care } & 191 & 12 & 12.2 & 17 & 18.3 & - \\
\hline \multicolumn{2}{|c|}{ Duration since first symptom/onset (mean days (SD)) } & & \multicolumn{2}{|c|}{$6(4)$} & \multicolumn{2}{|c|}{$5(5)$} & - \\
\hline \multirow{2}{*}{$\begin{array}{l}\text { Residence } \\
\text { preceding } \\
\text { admission }\end{array}$} & AL/Rehab/OSH/NH & & 12 & 11.8 & 56 & 59.6 & $<0.001$ \\
\hline & Home & & 90 & 88.2 & 38 & 40.4 & $<0.001$ \\
\hline \multicolumn{2}{|c|}{ Total comorbidities for patient, mean (SD) } & & \multicolumn{2}{|c|}{$4.0(2.0)$} & \multicolumn{2}{|c|}{$3.8(1.8)$} & - \\
\hline \multicolumn{2}{|l|}{ Dementia } & & 1 & 1.0 & 24 & 25.5 & $<0.001$ \\
\hline \multicolumn{2}{|c|}{ Chronic cardiac disease } & & 28 & 27.5 & 39 & 41.5 & 0.038 \\
\hline \multicolumn{2}{|c|}{ Chronic vascular disease } & & 9 & 8.8 & 19 & 20.2 & 0.023 \\
\hline \multicolumn{2}{|c|}{ Recurrent serious bacterial infection } & & 1 & 1.0 & 7 & 7.4 & 0.022 \\
\hline \multirow{2}{*}{$\begin{array}{l}\text { Pre-admission } \\
\text { medications }\end{array}$} & Antidepressants & & 9 & 8.8 & 19 & 20.2 & 0.023 \\
\hline & Antipsychotics & & 5 & 4.9 & 13 & 13.8 & 0.031 \\
\hline \multicolumn{2}{|c|}{ Has medical decision-making capacity } & & 81 & 79.4 & 34 & 36.2 & $<0.001$ \\
\hline \multicolumn{2}{|c|}{ Altered mental status } & & 7 & 6.9 & 28 & 29.8 & $<0.001$ \\
\hline Fall & & & 2 & 2.0 & 11 & 11.7 & 0.016 \\
\hline Body mass inde & $\mathrm{x}$, mean $(\mathrm{SD})$ & 177 & 28.4 & & 26. & & 0.007 \\
\hline Survived & $(\mathrm{BMI}<23)$ & 37 & 9 & 37.5 & 15 & 62.5 & 0.033 \\
\hline & (BMI 23-30) & 93 & 36 & 60.0 & 24 & 40.0 & - \\
\hline & $(\mathrm{BMI}>30)$ & 47 & 19 & 56.6 & 49 & 43.4 & - \\
\hline $\begin{array}{l}\text { Lowest oxygen } \\
\text { saturation }\end{array}$ & $<90 \%$ & 193 & 57 & 55.9 & 46 & 50.5 & - \\
\hline Fever & Temp > 100.4 & & 34 & 33.3 & 18 & 20.5 & 0.047 \\
\hline oxygen type on & Room air & 190 & 20 & 20.0 & 24 & 26.7 & - \\
\hline & Nasal cannula or venturi-mask & & 55 & 55.0 & 30 & 33.3 & 0.003 \\
\hline & Non-rebreather & & 8 & 8.0 & 24 & 26.7 & 0.001 \\
\hline & Mechanical ventilation & & 17 & 17.0 & 12 & 13.3 & - \\
\hline initial & Discharged from Emergency Room & & 4 & 3.9 & 5 & 5.3 & - \\
\hline admission & General ward & & 70 & 68.6 & 64 & 68.1 & - \\
\hline & Intensive care & & 22 & 21.6 & 22 & 23.4 & - \\
\hline Initial chest & Clear lung fields & & 8 & 8.0 & 15 & 19.0 & 0.029 \\
\hline X ray & Unilateral infiltrates & & 9 & 9.0 & 10 & 12.7 & - \\
\hline & Bilateral infiltrates & & 83 & 83.0 & 54 & 68.4 & 0.022 \\
\hline Medical & Azithromycin & & 80 & 78.4 & 51 & 54.3 & $<0.001$ \\
\hline & Hydroxychloroquine & & 84 & 82.4 & 53 & 56.4 & $<0.001$ \\
\hline & Steroids (non-pulse) & & 44 & 43.1 & 23 & 24.5 & 0.006 \\
\hline & Pulse dose steroids & & 23 & 22.5 & 14 & 14.9 & - \\
\hline
\end{tabular}


Table I. Cont.

\begin{tabular}{|c|c|c|c|c|c|c|c|}
\hline \multirow[t]{2}{*}{ Parameter } & & \multirow[t]{2}{*}{$N$} & \multicolumn{2}{|c|}{ Age 65 to 76 years } & \multicolumn{3}{|c|}{ Age 77 years and older } \\
\hline & & & $N=102$ & $\%$ & $N=94$ & $\%$ & $P$-value \\
\hline \multirow{4}{*}{$\begin{array}{l}\text { Medical } \\
\text { treatments }\end{array}$} & Ritonavir & & 6 & 5.9 & 4 & 4.3 & - \\
\hline & Saquinavir & & 3 & 2.9 & 0 & 0.0 & - \\
\hline & Tocilizumab & & 14 & 13.7 & 2 & 2.1 & 0.003 \\
\hline & Plasma & & 28 & 27.5 & 17 & 18.1 & - \\
\hline \multicolumn{2}{|c|}{ Any COVID-19-directed medication } & & 87 & 85.3 & 71 & 75.5 & - \\
\hline \multicolumn{2}{|c|}{ Total Doses of COVID-19-directed medications (SD) } & & \multicolumn{2}{|c|}{$15.6(15.3)$} & \multicolumn{2}{|c|}{$7.4(7.9)$} & $<0.001$ \\
\hline \multirow[t]{5}{*}{ Disposition } & Death & & 27 & 26.5 & 39 & 41.5 & 0.026 \\
\hline & Home & & 56 & 54.9 & 28 & 29.8 & $<0.001$ \\
\hline & Other & & 10 & 9.8 & 7 & 7.4 & - \\
\hline & Rehab & & 0 & 0.0 & 4 & 4.3 & - \\
\hline & SNF & & 9 & 8.8 & 16 & 17.0 & - \\
\hline \multicolumn{2}{|c|}{ Length of hospital stay [days] mean (SD) } & & \multicolumn{2}{|c|}{$16.4(18.6)$} & \multicolumn{2}{|c|}{$11.2(12.0)$} & 0.021 \\
\hline \multicolumn{2}{|c|}{ Required intensive care } & 80 & 45 & 44.1 & 35 & 37.2 & - \\
\hline \multicolumn{2}{|c|}{ Intensive care, survived $(\mathrm{LR}=1.9) \chi^{2}=1.9$} & & $21 / 45$ & 47 & $11 / 35$ & 31 & 0.168 \\
\hline \multicolumn{2}{|c|}{ Required mechanical ventilation } & 65 & 35 & 34.3 & 30 & 31.9 & 0.722 \\
\hline \multicolumn{2}{|c|}{ Survived $(L R=3.0) \chi^{2}=3.0$} & & $16 / 35$ & 46 & $6 / 30$ & 20 & 0.029 \\
\hline \multirow{3}{*}{$\begin{array}{l}\text { Respiratory } \\
\text { status and } \\
\text { course }\end{array}$} & Oxygen requirements decreased & & 58 & 63.0 & 23 & 45.1 & 0.038 \\
\hline & Oxygen requirements increased & & 20 & 21.7 & 15 & 29.4 & - \\
\hline & Oxygen requirements unchanged & & 14 & 15.2 & 13 & 25.5 & - \\
\hline $\begin{array}{l}\text { Required } \\
\text { vasopressors }\end{array}$ & Total & 58 & 32 & 31.4 & 26 & 27.7 & 0.569 \\
\hline \multirow{2}{*}{$\begin{array}{l}\text { Vasopressors } \\
\text { and course }\end{array}$} & No longer needed & & $14 / 32$ & 43.8 & $8 / 26$ & 30.8 & - \\
\hline & Needed to death/DC & & $18 / 32$ & 56.3 & $18 / 26$ & 69.2 & - \\
\hline \multicolumn{2}{|c|}{ Vasopressors, survived $(L R=4.6) \chi^{2}=4.4$} & & $13 / 32$ & 41 & $4 / 26$ & 15 & 0.046 \\
\hline \multirow{3}{*}{$\begin{array}{l}\text { Delirium and } \\
\text { course }\end{array}$} & Total & 82 & 32 & 31.4 & 50 & 53.2 & 0.002 \\
\hline & Delirium resolved & & $14 / 32$ & 43.8 & $7 / 50$ & 14.0 & 0.003 \\
\hline & Delirium remained or worsened & & $18 / 32$ & 56.3 & $43 / 50$ & 86.0 & \\
\hline \multicolumn{2}{|c|}{ Delirium survived $(L R=268) \chi^{2}=0.268$} & & $14 / 32$ & 43.8 & $19 / 50$ & 38.0 & 0.604 \\
\hline \multirow{4}{*}{$\begin{array}{l}\text { Hepatic } \\
\text { dysfunction } \\
\text { and course }\end{array}$} & Total & 83 & 54 & 52.9 & 29 & 30.9 & 0.002 \\
\hline & Hepatic function improved & & 23 & 42.6 & 8 & 27.6 & - \\
\hline & Hepatic function remained abnormal & & 12 & 22.2 & 14 & 48.3 & 0.015 \\
\hline & $\begin{array}{l}\text { hepatic functioned worsened until } \\
\text { death or DC }\end{array}$ & & 19 & 35.2 & 7 & 24.1 & - \\
\hline \multicolumn{2}{|c|}{ Hepatic dysfunction, survived $(L R=12.9) \chi^{2}=12.5$} & & $35 / 54$ & 65 & $7 / 29$ & 24 & $<0.001$ \\
\hline \multirow[t]{4}{*}{ Renal failure } & Total & 81 & 43 & 42.2 & 38 & 40.4 & 0.806 \\
\hline & $\begin{array}{l}\text { Renal function remained abnormal, } \\
\text { No RRT needed }\end{array}$ & & 4 & 9.3 & 16 & 42.1 & 0.001 \\
\hline & Worsened, RRT needed & & 19 & 44.2 & 15 & 39.5 & - \\
\hline & Improved, no RRT needed & & 20 & 46.5 & 7 & 18.4 & 0.007 \\
\hline Renal failure, & Irvived $(L R=6.03) \chi^{2}=5.9$ & & 24 & 56 & 11 & 29 & 0.015 \\
\hline Required rena & eplacement therapy & 34 & 19 & 100 & 15 & 100 & - \\
\hline RRT, survived & $=1.49 \chi^{2}=1.38$ & & $4 / 19$ & 21 & $1 / 15$ & 6.7 & $0.355^{*}$ \\
\hline
\end{tabular}

AL - Assisted Living Facility, DC - discharge, NH - long-term nursing care facility, OSH - Outside Hospital, Rehab - Rehabilitation Facility, $S D$ - standard deviation, $R R T$ - renal replacement therapy, $L R$ - likelihood ratio. 
Table III. Presenting factors associated with survival to discharge

\begin{tabular}{|c|c|c|c|c|c|c|c|c|c|c|c|}
\hline \multirow[t]{3}{*}{ Parameter } & \multicolumn{8}{|c|}{ Univariate } & \multirow{2}{*}{\multicolumn{3}{|c|}{ Regression }} \\
\hline & \multicolumn{3}{|c|}{ Lived } & \multicolumn{5}{|c|}{ Died } & & & \\
\hline & Count & $\%$ & $95 \% \mathrm{Cl}$ & Count & $\%$ & $95 \% \mathrm{Cl}$ & $\chi^{2}$ & $P$-value & $\begin{array}{l}\text { Odds } \\
\text { Ratio }\end{array}$ & $95 \% \mathrm{Cl}$ & $P$-value \\
\hline Age 65 to 76 & $75 / 130$ & 58 & $(49-66)$ & $27 / 66$ & 41 & $(30-53)$ & 4.9 & 0.026 & 2.1 & $(0.42-10)$ & 0.370 \\
\hline $\begin{array}{l}\text { Age } 77 \text { and } \\
\text { older [years] }\end{array}$ & $55 / 130$ & 42 & $(34-51)$ & $39 / 66$ & 59 & $(47-70)$ & & & & & \\
\hline Myalgia & $27 / 130$ & 21 & $(15-28)$ & $2 / 66$ & 3 & $(0.6-9)$ & 10.9 & 0.001 & 11.6 & $(1.1-127)$ & 0.044 \\
\hline $\begin{array}{l}\text { Has medical } \\
\text { decision- } \\
\text { making } \\
\text { capacity }\end{array}$ & $88 / 130$ & 68 & $(59-75)$ & $27 / 66$ & 41 & $(30-53)$ & 15.1 & $<0.001$ & 5.5 & $(1.0-30)$ & 0.051 \\
\hline $\begin{array}{l}\text { Procalcitonin } \\
\text { elevated }\end{array}$ & $13 / 84$ & 16 & $(9-24)$ & $25 / 39$ & 64 & (49-78) & 29.5 & $<0.001$ & 9.8 & $(2.4-40)$ & 0.002 \\
\hline $\begin{array}{l}\text { Lowest } \\
\mathrm{O}_{2}<90 \%\end{array}$ & $59 / 128$ & 46 & $(38-55)$ & $44 / 65$ & 68 & $(56-78)$ & 8.1 & 0.004 & 3.4 & $(0.78-14)$ & 0.104 \\
\hline $\begin{array}{l}\text { AST } \geq 2 x \\
\text { upper normal }\end{array}$ & $20 / 120$ & 17 & $(11-24)$ & $26 / 66$ & 39 & $(28-51)$ & 11.8 & 0.001 & 1.3 & $(0.32-5)$ & 0.734 \\
\hline $\begin{array}{l}\text { White blood } \\
\text { count elevated }\end{array}$ & $16 / 122$ & 13 & $(8-20)$ & $30 / 66$ & 46 & $(34-57)$ & 24.2 & $<0.001$ & 5.3 & $(1.3-21)$ & 0.018 \\
\hline $\begin{array}{l}\text { D-dimer } \geq 5 \times \\
\text { upper normal }\end{array}$ & $20 / 99$ & 20 & $(13-29)$ & $32 / 59$ & 54 & $(42-67)$ & 19.4 & $<0.001$ & 4.2 & $(1.1-16)$ & 0.034 \\
\hline $\begin{array}{l}\text { SSRI/antide- } \\
\text { pressant }\end{array}$ & $12 / 130$ & 9 & $(5-15)$ & $16 / 66$ & 24 & $(15-36)$ & 8.1 & 0.005 & 5.6 & $(0.89-35)$ & 0.066 \\
\hline $\begin{array}{l}\text { Altered mental } \\
\text { status }\end{array}$ & $18 / 130$ & 14 & $(9-21)$ & $17 / 66$ & 26 & $(16-37)$ & 4.2 & 0.040 & 8.1 & $(0.97-67)$ & 0.054 \\
\hline Dementia & $10 / 130$ & 8 & $(4-13)$ & $15 / 66$ & 23 & $(14-34)$ & 8.9 & 0.003 & 1.7 & $(0.08-41)$ & 0.728 \\
\hline
\end{tabular}

logistic regression analysis, $2 x-2$ times, $5 x-5$ times, Lowest $\mathrm{O}_{2}$-oxygen saturation recorded on room air and lower than $90 \%$.

but was significantly lower for those 77 years of age and above. Our findings are consistent with earlier studies which have shown that patients in the higher age groups, particularly those over age 80 years, had a significantly greater risk of death compared to younger patients [1, 2, 6-10]. We did not observe differences in survival related to hypertension, diabetes, cardiovascular disease, or total number of medical comorbidities, which have been described in prior reports [11-13]. Body mass index was not associated with differences in survival except that survival was higher among older compared to old patients with low BMI $\left(<23 \mathrm{~kg} / \mathrm{m}^{2}\right)$. The proportions of patients who received advanced therapies (e.g. haemodialysis, mechanical ventilation) did not differ significantly by age group. However, the total number of per-patient doses of COVID-19-directed medications was lower among older patients. Survival was similar for patients receiving most medications, except that those who received corticosteroids had lower survival. This was probably due to greater use of steroids among the sickest patients, a pattern that shifted to general use in the later pandemic following publication of the Randomised Evaluation of COVID-19 Therapy (RECOVERY) trial [14].
Overall lower medication use in the older group may have been due to factors such as patient/ family preferences or higher potential for medication side effects in the older age group.

Of note, survival was lower for older versus old patients who received mechanical ventilation (66\% vs. $34 \%$ ). In prior studies, mortality was $97 \%$ for those older than 65 years who received mechanical ventilation, and in a meta-analysis that included over 50,000 patients mortality was $84 \%$ for those age $>80$ years $[1,15]$. These differences may have been related to factors such as inclusion of patients who remained hospitalized, differences in steroid use, or intubation strategies.

Identification of prognostic, clinical, and laboratory indicators on admission and throughout the course of hospitalisation can assist provider discussions with COVID-19 patients and their families. We found a number of presenting factors including dementia [16], new loss of decision-making capacity, depression, myalgia, and hypoxia [17], and elevations in inflammatory markers, total white blood cell count, and procalcitonin to be associated with reduced survival in univariate analysis. In a prior meta-analysis, liver function-related tests and inflammatory mark- 
ers were among the most important mortality predictors [13]. After adjusting for age and other factors, we found that even modest elevations of AST and D-dimer levels or total white blood cell count were independent predictors of mortality. Elevations in total white blood cell count in COVID-19 were found to be predictive of mortality in another recent study [18]. Worsening clinical status, such as the need for mechanical ventilation or pressor support or the development of acute kidney injury or new hepatic dysfunction, increased mortality 2-3-fold in older versus old patients in this study. Conversely, in both older and old patients, improving mentation, oxygenation, as well as hepatic and renal function were highly significant and favourable prognostic indicators. In our cohort, around 90\% of patients for whom these clinical indicators were improving during hospitalization survived to discharge.

The prevalence of atypical symptoms among patients aged 65 years and over reaffirms the need for physicians to have a high index of suspicion for COVID-19 diagnosis. Almost half of all patients had no fever or cough, and over one-third did not complain of shortness of breath. Atypical symptoms such as anorexia, altered mental status, and generalized weakness were common. In addition, older patients were less likely to be diagnosed with COVID-19 as the primary diagnosis on admission. They also presented with more subtle symptoms initially than their younger counterparts, including lack of fever and normal CXR. In addition, the older patients were more likely to have comorbidities such as dementia, and chronic cardiac and vascular disease, which can obscure COVID 19 symptoms.

In a large meta-analysis, the only symptom that was associated with decreased survival in hospitalized COVID-19 patients was dyspnoea [13]. Consistent with a recent report, we found that that hypoxaemia on admission (rather than respiratory symptoms) predicted poor outcome [17]. We also found that although myalgia was uncommon among the oldest patients, its presence was significantly associated with mortality - even after adjusting for other factors. It is plausible that muscle pain may represent a previously unrecognized, early marker of severe disease.

Consistent with earlier reports, we found that dementia was associated with higher mortality in a univariate analysis [16, 19-21]. This association, however, was less clear when other factors were considered as well. Patients with dementia appear to be more easily infected and harmed by SARS-CoV-2 than those with normal mental function [16]. It has been speculated that pre-existing brain damage may permit greater viral entry into the nervous system, which is worsened by hypoxia and other organ failure. On admission, over one-third of older patients in this study lacked decision-making capacity due to underlying dementia and/or delirium. Older patients were also more likely to change their advance care directives/ resuscitation wishes during their hospitalization. The potential for rapid mental decline in the setting of COVID-19 underscores the need for patients and their families to proactively anticipate circumstances in which surrogate decision-making could be necessary, and to timely craft suitable advanced directives [22, 23].

An important concern during the pandemic has been the possibility that members of racial and ethnic minority groups have been disproportionately impacted. Racial disparities in terms of hospitalizations and death due to COVID-19 have been reported $[24,25]$. In the present study, the highest number of COVID-19 admissions were among white $(66 \%)$ patients followed by Hispanic $(17 \%)$, black (11\%), and Asian/Pacific Islander (4\%). We did not observe significant differences in survival across racial and ethnic groups (black $29 \%$, Hispanic $28 \%$, white $35 \%$, Asian 36\%).

This study had several limitations. First, this was a single-centre, retrospective cohort study with a relatively small sample size, which could influence the generalizability of results. Secondly, practice guidelines and patterns during the COVID-19 pandemic have continued to evolve. Such changes could render certain results less applicable to patients currently hospitalized with COVID-19. While the factor of age over or under 77 years was associated with higher mortality in a univariate analysis, after adjustment for other laboratory and clinical factors it was no longer significantly related to mortality. These findings suggest that other factors must be taken into account with age in order to more accurately predict mortality. Given the modest sample size, these results will require confirmation in larger studies.

In conclusion, among patients aged 65 years and older, age and other factors should be considered together to estimate the risk of mortality.

\section{Conflict of interest}

The authors declare no conflict of interest.

\section{References}

1. Richardson S, Hirsch JS, Narasimhan M, et al. Presenting characteristics, comorbidities, and outcomes among 5700 patients hospitalized with COVID-19 in the new york city area. JAMA 2020; 323: 2052-9.

2. Lewnard JA, Liu VX, Jackson ML, et al. Incidence, clinical outcomes, and transmission dynamics of severe coronavirus disease 2019 in california and washington: prospective cohort study. BMJ 2020; 369: m1923. 
3. Liu Y, Mao B, Liang S, et al. Association between age and clinical characteristics and outcomes of COVID-19. Eur Respir J 2020; 55: 2001112.

4. Zhou F, Yu T, Du R, et al. Clinical course and risk factors for mortality of adult inpatients with COVID-19 in Wuhan, China: a retrospective cohort study. Lancet 2020; 395: 1054-62.

5. Kang SJ, Jung SI. Age-related morbidity and mortality among patients with COVID-19. Infect Chemother 2020; 52: $154-64$

6. Docherty $\mathrm{AB}$, Mulholland $\mathrm{RH}$, Lone $\mathrm{NI}$, et al. Changes in in-hospital mortality in the first wave of COVID-19. a multicentre prospective observational cohort study using the WHO clinical characterisation protocol UK. Lancet Respir Med 2021; 9: 773-85.

7. Bonanad C, Garcia-Blas S, Tarazona-Santabalbina F, et al. The effect of age on mortality in patients with COVID-19: a meta-analysis with 611,583 subjects. J Am Med Dir Assoc 2020; 21: 915-8.

8. Yanez ND, Weiss NS, Romand JA, Treggiari MM. COVID-19 mortality risk for older men and women. BMC Public Health 2020; 20: 1742.

9. Du RH, Liang LR, Yang CQ, et al. Predictors of mortality for patients with COVID-19 pneumonia caused by SARS-CoV-2: a prospective cohort study. Eur Respir J 2020; 55: 2000524.

10. Nabors C, Sridhar A, Hooda U, et al. Characteristics and outcomes of patients 80 years and older hospitalized with coronavirus disease 2019 (COVID-19). Cardiol Rev 2021; 29: 39-42.

11. Saifi ES, Giorgi-Pierfranceschi M, Salvetti M, Maninetti L, Cavalli I, Muiesan ML. Factors associated with survival in older patients affected by COVID-19: a retrospective cohort study. Arch Gerontol Geriatr 2021; 94 104349.

12. Mehraeen E, Karimi A, Barzegary A, et al. Predictors of mortality in patients with COVID-19-a systematic review. Eur J Integr Med 2020; 40: 101226.

13. Mesas AE, Cavero-Redondo I, Alvarez-Bueno C, et al. Predictors of in-hospital COVID-19 mortality: a comprehensive systematic review and meta-analysis exploring differences by age, sex and health conditions. PLoS One 2020; 15: e0241742.

14. RECOVERY Collaborative Group, Horby P, Lim WS, et al. Dexamethasone in hospitalized patients with covid-19. N Engl J Med 2021; 384: 693-704

15. Lim ZJ, Subramaniam A, Ponnapa Reddy M, et al. Case fatality rates for patients with COVID-19 requiring invasive mechanical ventilation. A meta-analysis. Am J Respir Crit Care Med 2021; 203: 54-66.

16. Wang Q, Davis PB, Gurney ME, Xu R. COVID-19 and dementia: analyses of risk, disparity, and outcomes from electronic health records in the US. Alzheimers Dement 2021; 17: 1297-306.

17. Chatterjee NA, Jensen PN, Harris AW, et al. Admission respiratory status predicts mortality in COVID-19. Influenza Other Respir Viruses 2021; 15: 569-72.

18. Feng $X$, Zhu B, Jiang C, et al. Correlation between white blood cell count at admission and mortality in COVID-19 patients: a retrospective study. Available at research square [https://Doi.org/10.21203/rs.3.rs-20383/v1]. 12 May 2020, PREPRINT (Version 1)

19. Graf CE, Herrmann FR, Spoerri A, et al. Impact of body composition changes on risk of all-cause mortality in older adults. Clin Nutr 2016; 35: 1499-505.

20. Covino M, De Matteis G, Polla DAD, et al. Predictors of in-hospital mortality AND death RISK STRATIFICATION among COVID-19 PATIENTS aged $>/=80$ YEARs OLD. Arch Gerontol Geriatr 2021; 95: 104383.

21. Hariyanto TI, Putri C, Arisa J, Situmeang RFV, Kurniawan A. Dementia and outcomes from coronavirus disease 2019 (COVID-19) pneumonia: a systematic review and meta-analysis. Arch Gerontol Geriatr 2021; 93: 104299.

22. Dhand A, Forman L, Vegunta R, Aronow WS, Nabors C. Challenges of shared decision making in older patients with COVID-19. J Am Geriatr Soc 2021; 69: 605-6.

23. Morandi A, Di Santo SG, Zambon A, et al. Delirium, dementia, and in-hospital mortality: the results from the italian delirium day 2016. A national multicenter study. J Gerontol A Biol Sci Med Sci 2019; 74: 910-6.

24. Price-Haywood EG, Burton J, Fort D, Seoane L. Hospitalization and mortality among black patients and white patients with covid-19. N Engl J Med 2020; 382: 2534-43.

25. Egede LE, Walker RJ. Structural racism, social risk factors, and Covid-19 - a dangerous convergence for black americans. N Engl J Med 2020; 383: e77. 